\title{
Microrobotic Streak Seeding For Protein Crystal Growth
}

\author{
Atanas Georgiev $^{1}$, Peter K. Allen ${ }^{1}$, Ting Song ${ }^{2}$, Andrew Laine ${ }^{2}$, William Edstrom ${ }^{3}$, John Hunt ${ }^{3}$ \\ Department of Computer Science ${ }^{1} /$ Biomedical Engineering ${ }^{2} /$ Biological Sciences $^{3}$ \\ Columbia University, New York, NY 10027 \\ \{atanas,allen\}@cs.columbia.edu, \{al418,ts2060\}@ columbia.edu \\ \{edstrom,jfhunt $\} @$ biology.columbia.edu
}

\begin{abstract}
We present a microrobotic system for protein crystal micromanipulation tasks. The focus in this paper is on the task known to crystallographers as streak seeding - it is used to entice certain protein crystals to grow. Our system features a set of custom designed micropositioner end-effectors we call microshovels to replace traditional tools used by crystallographers for this task. We have used micro-electrical mechanical system (MEMS) techniques to design and manufacture various shapes and quantities of microshovels. Visual input from a camera mounted on the microscope is used to detect the locations of the source crystals which the tool needs to touch as well as the locations of the target protein droplets for seeding. We present experimental results that illustrate the applicability of our approach.
\end{abstract}

\section{INTRODUCTION}

The completion of the human genome has led to a shift in biologists' focus toward proteins, the product of genes. A large coordinated effort is under way worldwide to master the process of determining the 3-D atomic-level structure of proteins [1]. These experimentally determined structures can be combined with the sequence data using bioinformatics methods to produce structural coverage of the majority of proteins. This is expected to have a significant impact on biological and medical research by shedding light on the relationships of structure to function and disease, directing structure-based drug design [2] and refining our understanding of evolutionary relationships between proteins.

The structure determination effort centers on developing the capability of processing proteins on a large scale - a highthroughput pipeline starting from producing the proteins and leading through various stages to the eventual discovery of the spatial arrangement of the protein atoms. This necessitates the development of strategies and tools for automated and fast manipulation of protein crystals, as one of the most popular methods for structure determination is X-ray crystallography [3].

Automated protein crystal manipulation is a challenging task because of the unique combination of factors involved. Protein crystals are small (sizes of interest are between 25 and $1000 \mu \mathrm{m}$ ) and fragile. They are also sensitive to environmental variations, especially temperature. Their growth environment is a $0.1-5 \mu \mathrm{l}$ droplet of liquid which dehydrates in a matter of minutes once exposed to room conditions. Currently, the

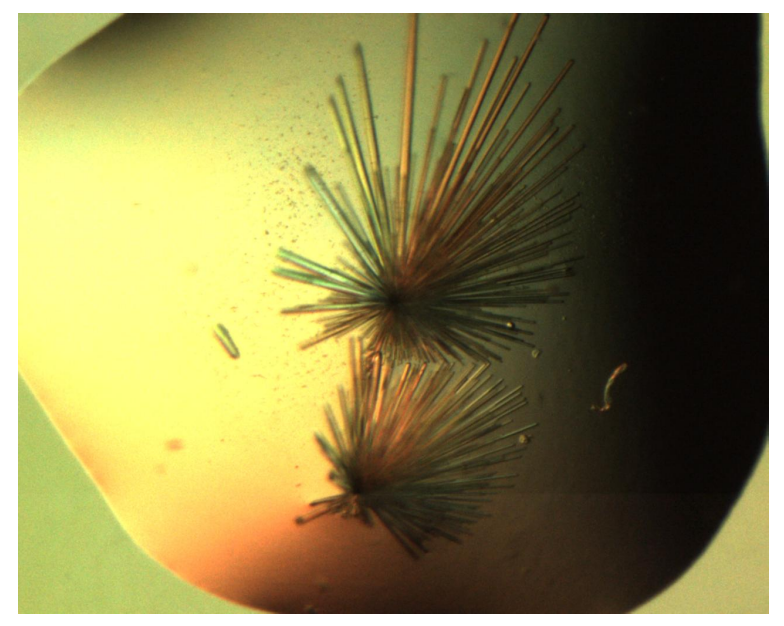

Fig. 1. Protein crystals.

mechanics of micromanipulation is poorly understood and thus results of sensorless micromanipulation strategies are unpredictable. Obtaining reliable feedback is also problematic.

In this paper, we describe a microrobotic system that will help to improve the throughput in crystal production. The focus here is on the task known by crystallographers as streak seeding - a technique where small nuclei (or microcrystals) are transferred to a protein solution that is optimized for their growth. In building high-throughput protein crystal production factories, optimizing crystal growth is a very key element. Streak seeding is normally performed using manual methods which are both slow and inefficient. Microrobotics can improve both the speed and the yields of the crystal production process.

In this work, we make use of microshovels - microtools we designed and manufactured out of silicon in various shapes and quantities using micro-electrical mechanical system (MEMS) techniques for protein crystal micromanipulation, including streak seeding. Visual input from a camera mounted on the microscope is used to detect the locations of the source crystals, which the tool needs to touch as well as the locations of the target droplets for seeding.

The remainder of this paper is organized as follows: After a brief review of the related work, we describe our hardware 
setup and the task at hand in Section III. In Section IV, we describe our methods for solving important problems in streak seeding automation. Experimental results are presented in Section V.

\section{RELATED WORK}

One of the major advances in robotics over the last 20 years is the use of visual sensing for robotic manipulation [4]. The advent of fast and inexpensive digital imaging technology has allowed camera systems to be integrated as part of a closedloop feedback control system [5]. Visual sensing and processing has been successfully implemented at the microscale level for manipulation of known micro-electromechanical systems with calibrated devices [6], [7] and has also been successfully used for biological cell injection [8].

In terms of the mechanics of the actual manipulation, quite a few very diverse ideas have been pursued. Various kinds of microgrippers have been proposed [9]. Optical trapping by a laser has been successfully used for both direct and indirect cell manipulation [10]. Surface tension is a very popular and safe way to handle small crystals [11]. A micromanipulation tool based on adhesive forces has been demonstrated [12]. Methods exploiting magnetic and electrostatic forces are also being used.

Unfortunately, not many of these approaches can be easily applied to our case. Microgrippers pose the risk of structural damage to the fragile crystals. Optical trapping has the potential to damage the crystals because of excessive heat. Adhesive forces are difficult to rely on because of the drastic variations of the composition of the protein crystals and their environments. In places where they can be used to collect micro-crystals, releasing these in the target solution may be a problem. It is not clear what the consequences to the crystals will be if dielectrophoresis is used.

Our approach is also different than what most current crystallographers use in that we use a non-organic solid material for seeding. Previous researchers have considered and tested cat whiskers, horse hair, rat whiskers, and dried seaweed, among others [13]. All of these are difficult and not straightforward to utilize on a microrobotic platform because they have been designed for manual use. Detecting them and tracking them in a closed-loop solution would be very challenging, while using them in a open-loop solutions would be extremely unreliable.

\section{SetUP AND TASK Description}

The task of streak seeding consists of transferring a multitude of small nuclei (micro-crystals) into a protein solution which will help them grow. This is often done when a researcher crystallographer has managed to cause the protein to nucleate under some conditions and now seeks to make the resulting crystals grow large enough to be used for X-ray data collection. Since the precise conditions for crystal growth are usually unknown and differ among the proteins, the researcher often performs the seeding many times, each on a slightly different solution in order to discover the one that produces

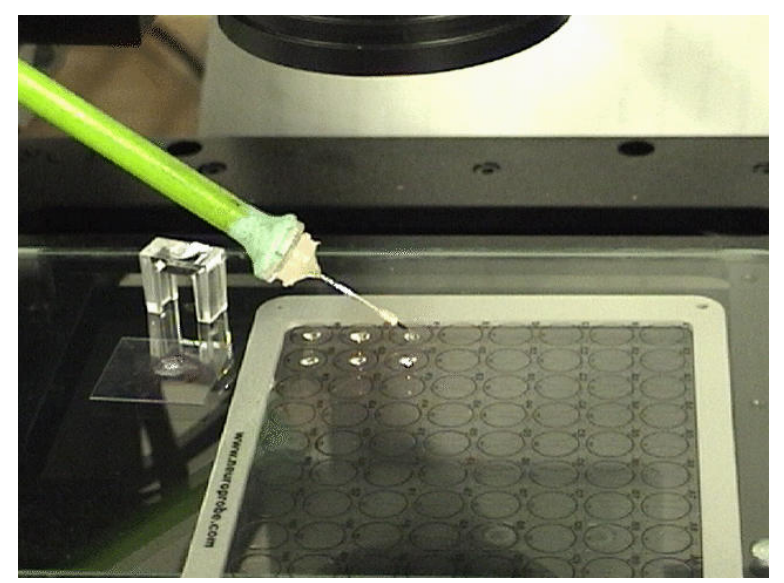

Fig. 2. Seeding six wells of a 96-well plate. The plastic slide on the left contains the source protein crystals. The small bucket behind it contains water for tool cleanup.

the optimal results. Thus the task inherently involves repetition and large quantities. It is normally carried out on standard 24well or 96-well plates (Figure 2).

Streak seeding is done manually by a lab technician who typically uses a whisker as a tool. There are two steps in the process: poking and streaking. First, the sharp edge of the whisker is used to poke a crystal residing in its original nucleation solution where it formed. The poking causes the whisker to break off and retain tiny microcrystals along its stem. Next, the technician streaks with the whisker through a small (about 2-3 $\mu l$ ) droplet of the new growth solution. This results in the transfer of some of the microcrystals from the whisker to the growth solution and their eventual enlargement if cared for properly.

Each of these droplets resides at its designated location on the plastic lid of the plate. Once seeding is completed, the lid is flipped over and depressed against the plate to hermetically seal each droplet into its own well in the plate. This prevents the crystals from dehydrating while stored away until they grow large.

Our goal in this project is to produce a microrobotic system that is capable of performing streak seeding autonomously. Our workstation (see Figure 3) is centered around a Sutter MP-285 micropositioner with three degrees of freedom (DOF) - independent motion control along the $\mathrm{X}, \mathrm{Y}$ and $\mathrm{Z}$ axes with accuracy as high as $40 \mathrm{~nm}$. A crystal manipulation tool (e.g. a cryogenic loop or a microshovel) is attached as the manipulator end-effector. The tool and the protein crystals are observed through an Olympus SZX12 optical microscope (eyeto-hand configuration [14]) which holds a 2-DOF motorized microscope stage capable of holding a 96-well plate and moving horizontally. The microscope provides a total magnification from $8.4 \mathrm{x}$ to $108 \mathrm{x}$ and has a CCD camera adapter onto which a Sony XC-77 camera is mounted. The camera, the micropositioner and the motorized stage are connected to a PC which processes the visual feedback and controls the actuators. 


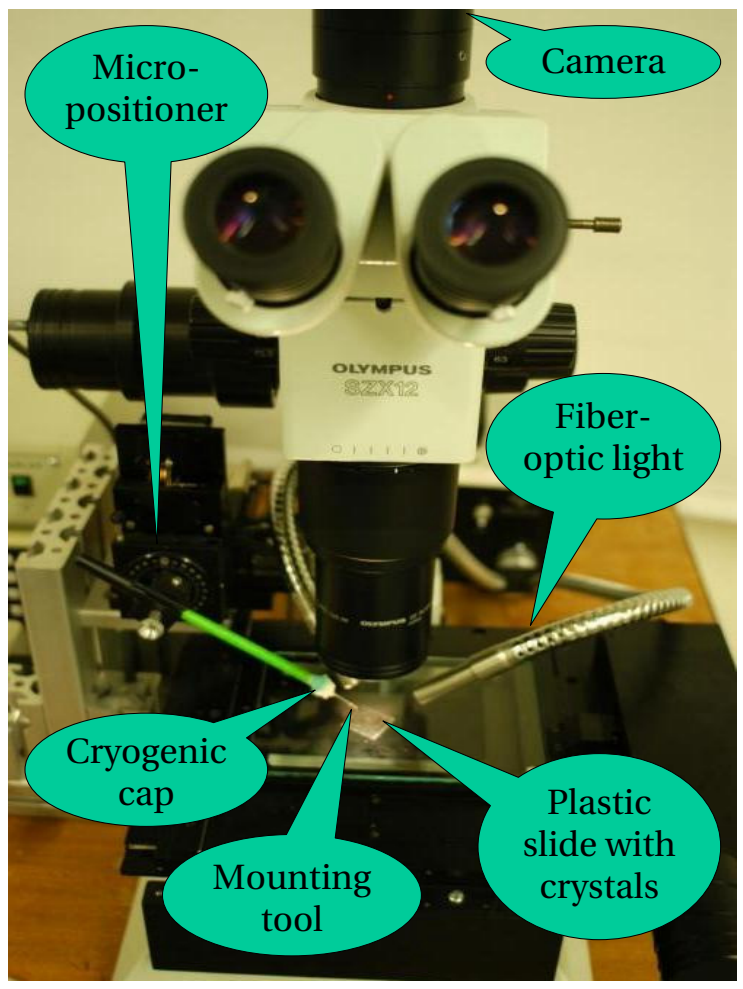

Fig. 3. Workstation for protein crystals manipulation.

At the beginning of the procedure, the user is asked to place the 96-well plate cover, a slide with the seeding crystals, and a bucket of water for tool cleaning at their designated locations. After that, the user clicks with the mouse and the system takes control.

\section{SEEDING}

Streak seeding is a complex task that can be broken down into a few smaller fundamental problems: choice of tools used, methods for detection of crystals in the visual feedback and methods for detection of the location of the seeding target droplet. These will be explained in order in the following subsections.

\section{A. Tools for seeding}

The type of manipulation tools used in a robotic application are among the very important factors that decide whether that application will be successful. The most popular tool for streak seeding among crystallographers is the cat whisker. Attempts have been also made to use pig bristles, horse hair, or thick human hair. These are all long and soft organic materials that are difficult to detect and track in an image and are extremely hard to use for object manipulation, be it on an open- or a closed-loop system.

In our system, we have addressed this issue by using our own custom tool, which we call a microshovel (Figure 4). We have designed and manufactured various quantities and sizes of microshovels out of silicon using methods employed by the MEMS industry.

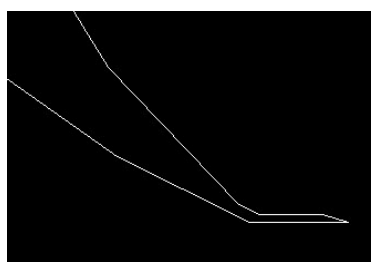

(a)

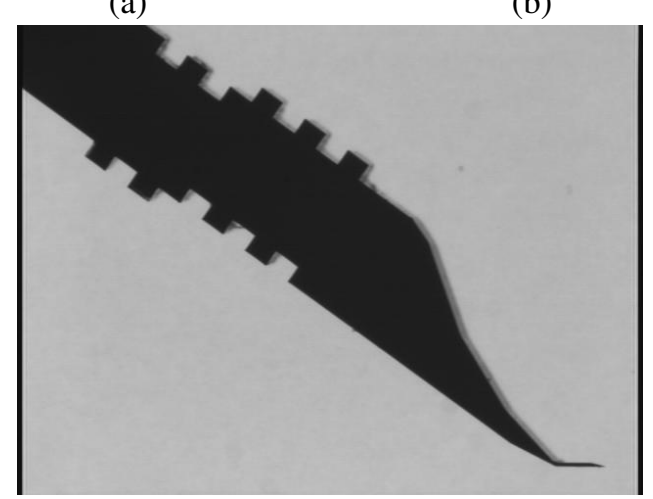

(c)

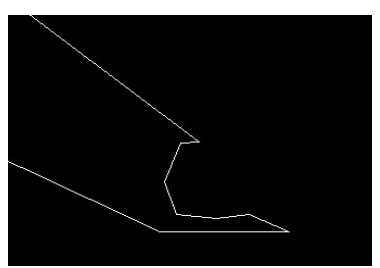

(b)
Fig. 4. Microshovels. (a) and (b) two sample design profiles. (c) a silicon microshovel.

In the design phase, we used a CAD package to draw the desired tool profiles (Figure 4(a) and (b)) and create the photomask pattern. The profiles differed mostly in their tips, with sizes ranging from 50 to $280 \mu \mathrm{m}$ in length and 7 to $40 \mu \mathrm{m}$ in height, as these covered entire expected range of protein crystal sizes we would manipulate. The square notches at the bottom of the tool stem encode the shape and the size of the tip, as it would otherwise be impossible to tell the microshovels apart by looking at them with a naked eye.

For manufacture, the mask pattern was transferred onto a $2 \times 2$-inch quartz photomask which was used to perform the photolithography on a 2-inch silicon wafer. The thickness of the wafer, which translated into the width of the tools, was $300 \mu \mathrm{m}$ and was, again, chosen so that it covers the expected range of crystal sizes. This wafer was bonded to a larger sacrificial wafer and the package underwent deep reactive ion etching (DRIE) through the entire small wafer leaving the tools only adherent to the larger one. Finally, the microshovels were detached and cleaned up from the bond and the photoresist in an acetone bath.

As may be obvious by Figure 4, we did not design and manufacture the microshovels for the sole purpose of streak seeding, but for the more general purpose of protein crystal manipulation. Our work is generally aimed at using computer vision to provide the compliance and robustness which precise crystal manipulation requires without the need for extensive analysis of the physics of grasping or a detailed knowledge of the environment. In a parallel effort [15], we are working on the automation of the task known as crystal mounting which consists of picking up an individual protein crystal from its growth solution for the purpose of X-ray data collection. Thus, the shapes of the microshovels have been also designed 


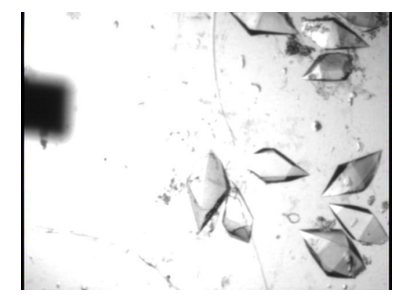

(a)

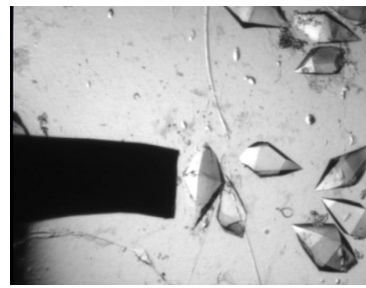

(c)

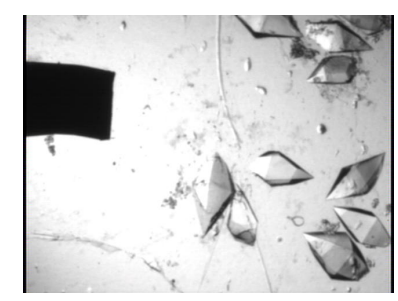

(b)

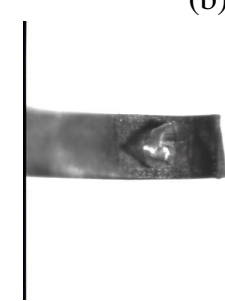

(d)
Fig. 5. A complete crystal mounting experiment: (a) the initial condition (b) the microshovel has descended into the droplet. (c) the microshovel has approached the target crystal. (d) the microshovel has picked up the crystal and raised it up above the liquid.

with crystal pickup in mind. An example shown in Figure 5 illustrates the use of a microshovel during the main stages of the task: First, the tool descends into the droplet with crystals under visual guidance (Figure 5b). Next, visual servoing is used to approach the target crystal with the tool (Figure 5c). Finally, the microshovel picks up the crystal by sliding under it and raising it up above the liquid (Figure 5d).

An interesting factor here become the different constraints imposed on the material by the two vastly different application tasks. On the one hand, it is essential for crystal mounting that the tool has very low background X-ray scattering, so that X-ray diffractograms obtained of a crystal mounted on a microshovel, are captured with as high an accuracy as possible. On the other hand, streak seeding demands that the microshovels have some rigid and retentive properties so that they break off and collect small fragments upon contact with a crystal, which are to be transferred to the clean protein solution. We have studied both phenomena and our results indicate that silicon indeed qualifies for both tasks. For streak seeding, it is at least on par with the current crystallographers' favorite - the cat whisker.

In terms of rigidity, silicon can be brittle, especially at the scales we are working at. However, this is not really an issue as the silicon is still much harder than the more fragile crystals we are handling. Additionally, the precision of our micropositioner is much higher than we really need, ensuring the accurate positioning of the tip. Our tests have shown that people with relatively normal motor skills can perform both streak seeding and crystal mounting manually without causing any structural damage to the tools; this is certainly not a problem for expert crystallographers and lab technicians.

\section{B. Crystal Detection}

It is extremely important to make a correct detection of the locations of the protein crystals used for seeding, as an incorrect guess will render the entire procedure useless. Unfortunately, the reliable detection of the protein crystals seems to be the most difficult part of the task. This is to a large extent because of the fact that different proteins form vastly different crystals in terms of their shape and size. This precludes, for example, the use of template-based matching algorithms.

We have established that edge-based detection approaches work best in our case. The algorithm is as follows:

1) Take an image of the crystals (Figure 6a) and apply an edge detector, for example using Sobel masks (Figure 6b)

2) Apply a morphological closure to the edge image to connect close nearby components and smooth up the contours. Follow with an opening operator to smooth the contours even further (Figure 6c). The structural element we use for both the opening and the closure is a small disk with a radius of a few pixels. It is not a good idea to use a larger disk because it may obliterate some, even many of the smaller crystals which may be about the same size.

3) Fill in the holes. For each background section (i.e. not edge) that is surrounded by an object, assign that background section to that object (Figure 6d).

4) Threshold the result into a binary image. We follow Otsu's method [16] for choosing the value of the threshold automatically based on minimizing the intensity variances of the two resulting classes (Figure 6e).

5) Remove the small objects from consideration (Figure 6f).

6) Label the remaining objects using connected component analysis.

The last step gives us the layout of the crystals on the image as captured by the camera on the microscope (Figure 6f). Since we have a calibrated system, we can compute these locations with respect to the micropositioner (modulo the depth of the slide where the crystals reside, which we know from calibration) and command it to go there.

We don't need to poke all crystals, one good contact usually suffices. We chose to use two randomly selected crystal segments to increase the robustness of the system in case of a false positive in the crystal detection.

\section{Droplet Detection}

We have a well-calibrated system in which most objects are positioned at known locations. The system uses hard-coded coordinates to position a certain object under the microscope. For example, from the origin, moving the stage 3 units to the left and 2 units down positions the center of well $\mathrm{C} 4$ directly under the microscope.

The protein droplets however are pipetted onto the tray manually and are subject to human accuracy constraints. They are not always deposited exactly at the center of the well and the offset can be large enough to make relying on hard-coded locations impractical. There is also another, practical problem. The magnification level of the microscope is not computer 


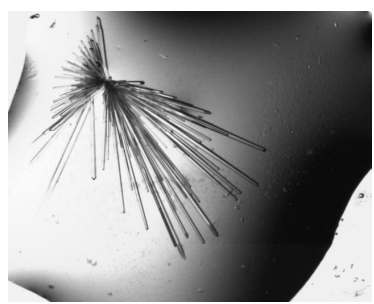

(a)

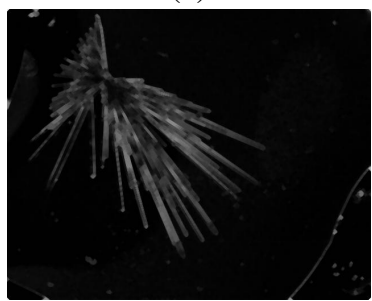

(c)

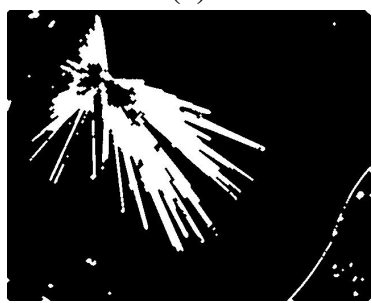

(e)

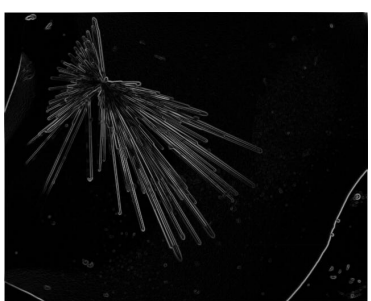

(b)

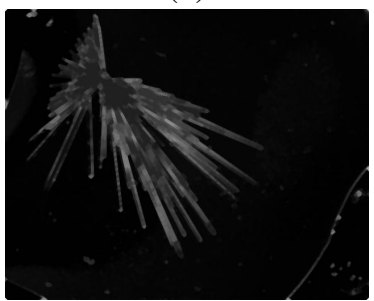

(d)

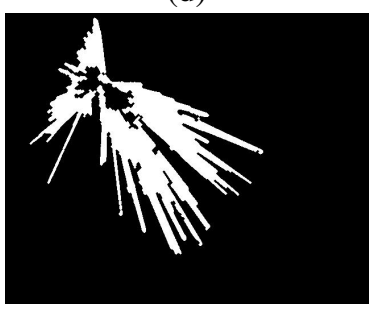

(f)
Fig. 6. Crystal detection steps. (a) Original image. (b) Detected edges. (c) Morphological closure and opening. (d) Holes filled in. (e) Thresholding. (f) Small objects removed.

controlled and requires us to fix it to a single common setting for all activities. This leads to some necessary tradeoffs: It is desirable to have a high enough magnification to be able to better detect the crystals for poking. On the other hand, this will make the field of view narrower, which for streaking means that the entire well will not be visible all at the same time.

Because of this and because of the manual deposition of the protein solution, the droplets of protein solution may be only partially visible to the camera during streaking when the well is moved directly under the microscope. Thus, we need to find the center of the droplet in order to perform the streaking successfully. Accuracy is not a major requirement for the center detection; the necessary condition is to make sure that the tool will streak through a large portion of the droplet.

Fortunately, the centers of the droplets are visually quite distinctive. This is because of the shape of the droplet and the way light rays propagate. Due to surface tension in the liquid, the droplet assumes a standard curved shape that acts as a light-scattering lens (Figure 7a). Light rays come from the microscope light below the stage. Those that pass near the center undergo very little refraction, if any, and pass through the droplet mostly undisturbed. On the other hand, the rays passing through closer to the periphery are being refracted away from the microscope objective. The result is a very clear
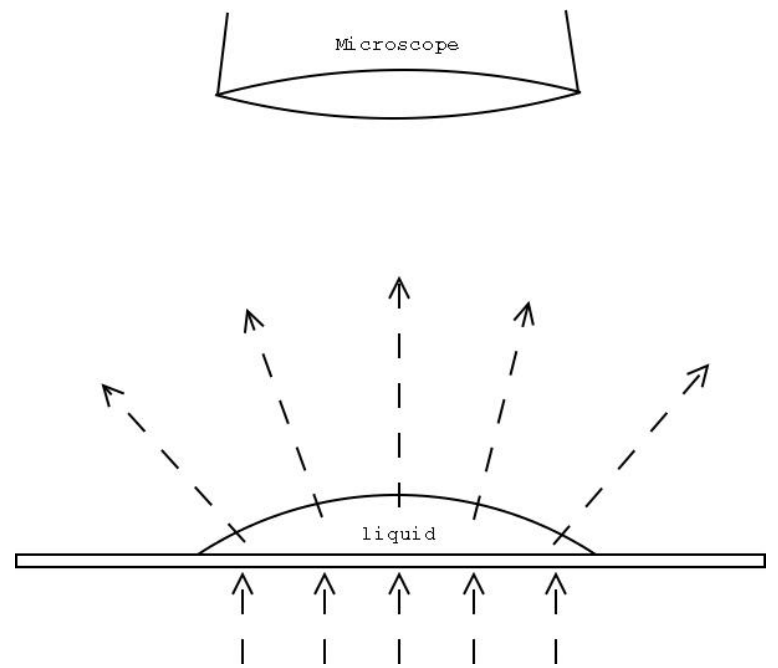

(a)

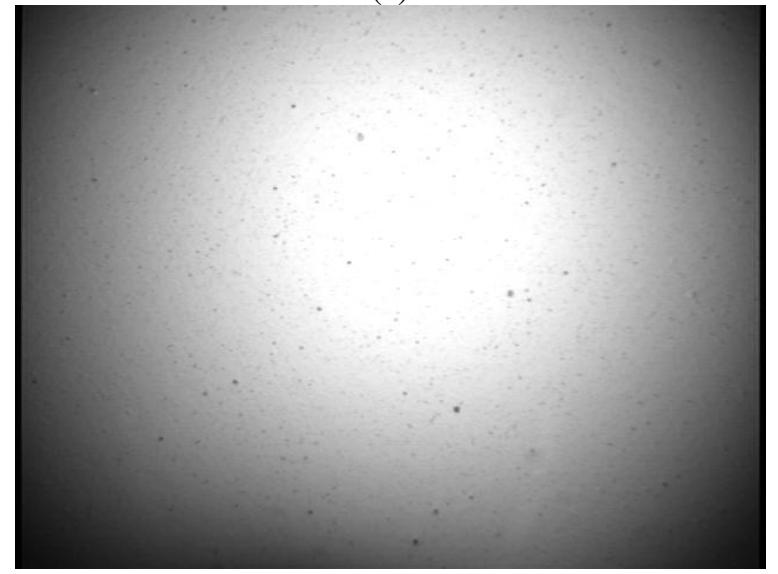

(b)

Fig. 7. Droplets. (a) Ray scattering by droplets. (b) a view of a droplet under microscope.

tapering of the intensity levels around the edges of the droplet (Figure 7b).

Thus, to find the center of the droplet is to find the position of its brightest spot. We do this, as follows:

1) Move microscope stage to hard-coded location such that the center of the target well is placed directly under the microscope optical axis. Acquire an image $I$.

2) Compute the intensity gradient of $I$. This is not necessary for every pixel in the image but could be done on a sample grid, say every 10 pixels. We use Sobel masks, so if $G x$ and $G y$ are the "measurements" of the two masks for location $[x, y]$, then $G=\sqrt{G x^{2}+G y^{2}}$ and $\theta=\arctan (G y / G x)$ are the magnitude and the direction of the gradient at this point.

3) Discard locations where the gradient is of very low magnitude ( $G$ is less than a certain threshold)

4) Use the rest of the computed gradients to vote in a Hough transform fashion for the center of the droplet (the peak or local maximum of the gradient). Each location votes for the line passing through it in the 


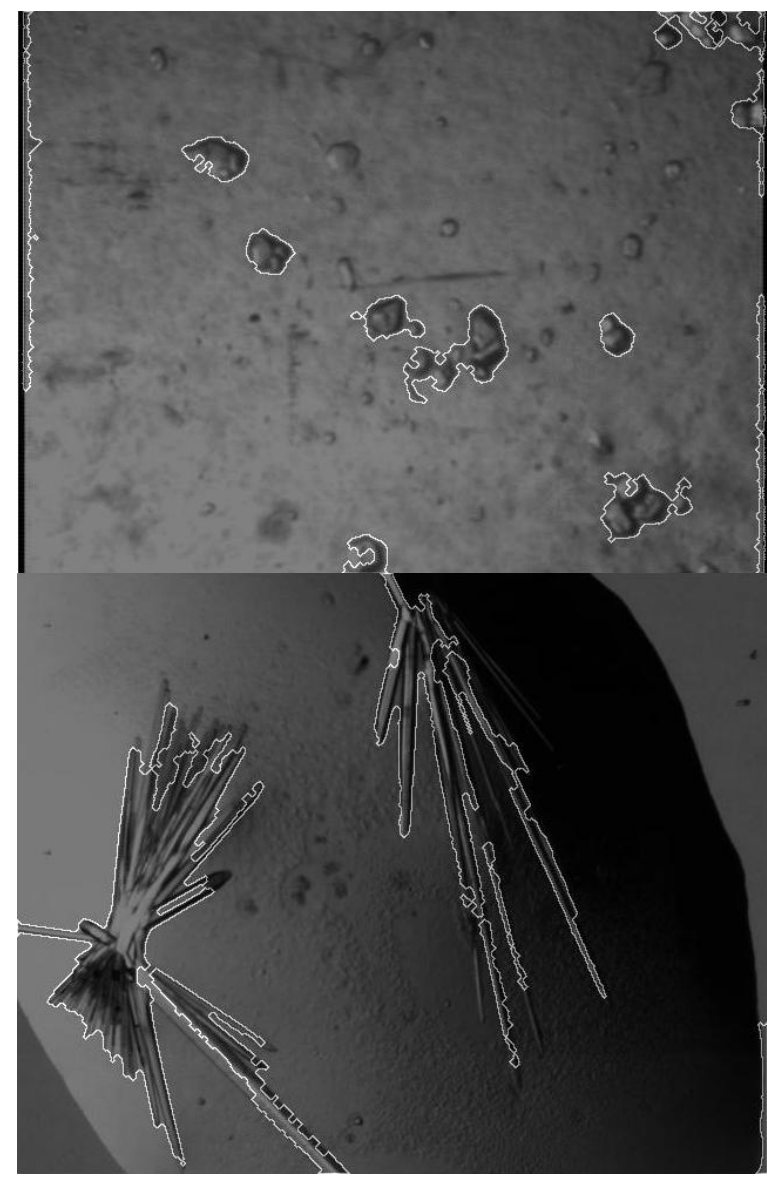

Fig. 8. Crystal detection examples. Detected crystals are surrounded by a white outline.

direction $\theta$ of the gradient.

5) Count the votes and find the peak. Knowing image-toreal-world coordinates from calibration, move the stage so the computed droplet center is under the microscope.

\section{EXPERIMENTS}

We have performed three kinds of tests to verify the viability of our methods. These tests were aimed at the three most important aspects of our system: the crystal detection, the droplet detection and their integration into a complete system.

First, we tested the ability of our crystal detection method. We ran the program on a set of images of real crystals we had acquired throughout our work in this area. Figure 8 shows two such results. As can be seen from the images, the algorithm correctly identified areas that are crystals (outlined in white). It should be noted that not all crystals were identified. However, in cases when ambiguities exist, it is better to err on the side of the false negatives, rather than accept the risk of false positives. As long as there are enough correctly identified crystals, the loss of few is not consequential.

The second test was directed at the computation of the correct location of a droplet. We ran a multitude of tests and the results were consistent: As long as there was enough portion of the droplet in the field of view, its center was
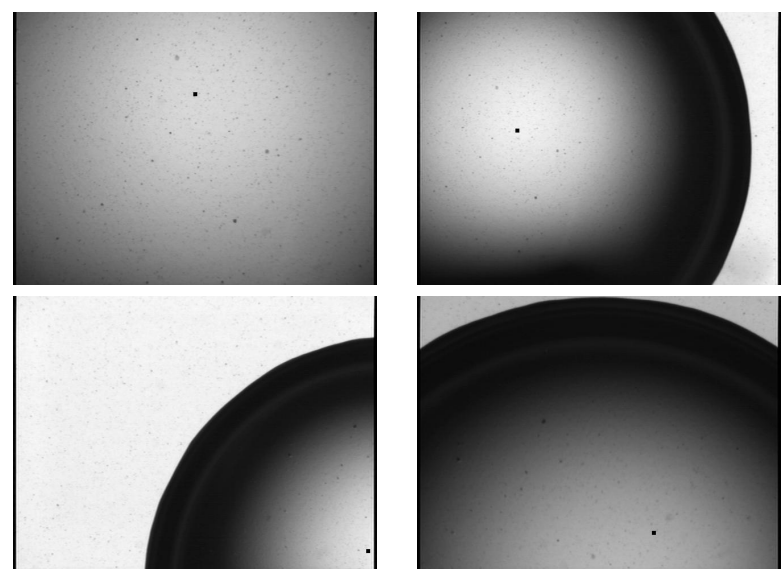

Fig. 9. Droplet detection examples. Computed droplet center is denoted by a small black square.

identified correctly. Here, we would like to point out that there is no need to seek the most possible accurate answer. It is simply sufficient to make sure that the stroking motion will go through a large portion of the droplet. Four sample results are shown in Figure 9. From there, we see that the method can be quite stable even with less than $25 \%$ of the droplet in the field of view (see bottom left run in Figure 9).

Finally, we tested the entire seeding system as a whole by giving it the task of performing seeding on actual crystals. Figures 10 and 11 show snapshots of the task execution on two wells. Each example starts with a snapshot of the seeding source with the crystals. The second image shows the result of the segmentation of the image into crystals (white) and background (black). The third and fourth images show the microshovel poking two different crystals. The fifth image is taken after the target well has been positioned under the microscope by the motorized stage. The black square indicates the estimated center of the droplet. Finally, the last image in the sequence shows the microshovel dipped and streaking through the target liquid.

These experiments illustrate that the system can be used successfully for real seeding. We have done so on standard 96-well Neuroprobe plates. Based on the rate of protein dehydration, which causes degradation of the streak seeding results and the efficiency of the image processing, our target time for the processing of one plate was set to 15 minutes. The test runs demonstrated that our system's performance fits into the allotted time frame and can complete the task in 14-15 minutes.

\section{COnClusion And Future Work}

We have presented a functional robotic system for streak seeding of protein crystals. It is based on a micropositioner, an optical microscope with a motorized stage and a CCD camera for visual feedback, and software control through a regular PC. The system features a set of newly developed and unconventional seeding tools custom designed and manufactured out of silicon using contemporary MEMS techniques. We also presented a number of computer vision and robotics 

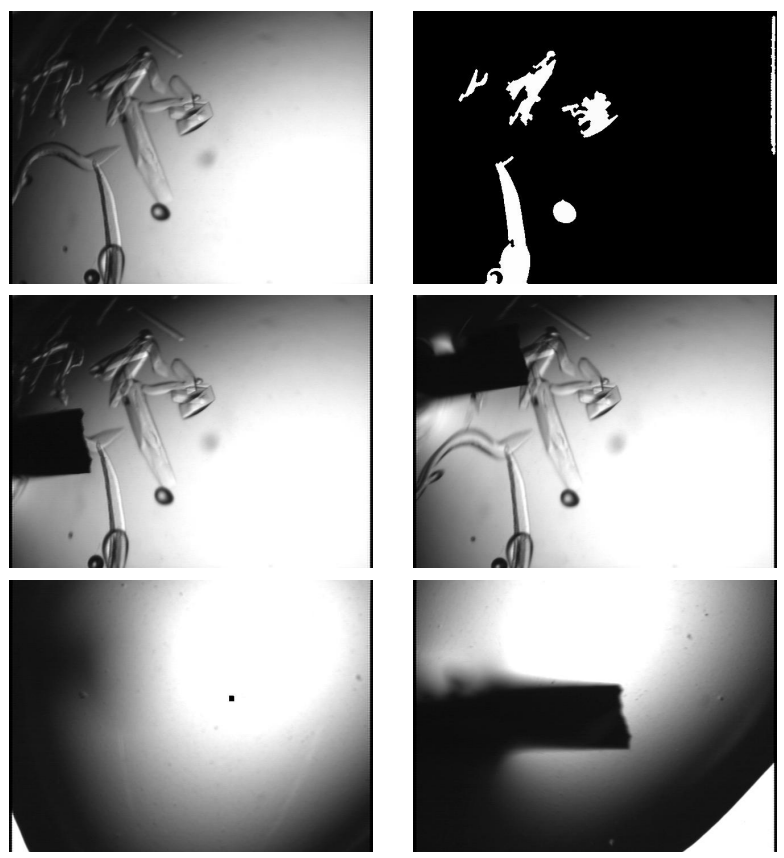

Fig. 10. Complete seeding example 1. (a) Initial view of the seeding crystals. (b) detected crystals (in white). (c) microshovel poking crystal 1. (d) microshovel poking crystal 2. (e) computed center of target droplet (marked with black square). (f) microshovel streaking through target droplet.

techniques that allow us to detect the location of the source crystals and the target droplet with sufficient accuracy for the task at hand.

The system is not completely foolproof as is any system relying on a single source of the complexity of a CCD camera. In the future, we would like to address its robustness in complex cases where impurities and precipitate are common factors affecting its performance.

\section{ACKNOWLEDGEMENT}

This work is partially supported by a grant to the Northeast Structural Genomics Consortium from the Protein Structure Initiative of the National Institute of Health (P50 GM62413).

\section{REFERENCES}

[1] NIH. (2004) The protein structure initiative. [Online]. Available: http://www.nigms.nih.gov/psi

[2] C. L. Verlinde, "Structure-based drug design: progress, results and challenges," Structure 2, pp. 577-587, 1994.

[3] V. L. Niennaber, P. Richardson, V. Klighover, J. Bouska, V. L. Giranda, and J. Geer, "Discovering novel ligands for macromolecules using x-ray crystallographic screening," Nat. Biotechnol., vol. 18, pp. 1105-1108, 2000.

[4] S. Hutchinson, G. Hager, and P. Corke, "A tutorial on visual servo control," IEEE Trans. on Robotics and Automation, vol. 12, no. 5, pp. 651-670, October 1996.

[5] P. K. Allen, A. Timcenko, Y. B., and P. Michelman, "Automated tracking and grasping of a moving object with a robotic hand-eye system," IEEE Trans. on Robotics and Automation, vol. 9, no. 2, pp. 152-165, April 1993.
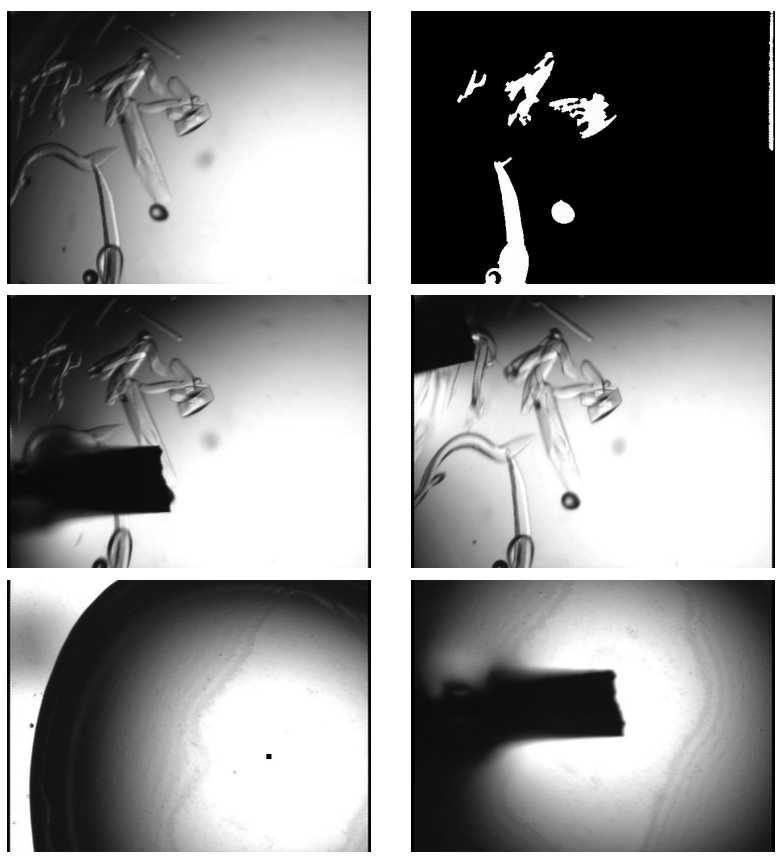

Fig. 11. Complete seeding example 2. (a) initial view of the seeding crystals. (b) detected crystals (in white). (c) microshovel poking crystal 1. (d) microshovel poking crystal 2. (e) computed center of target droplet (marked with black square). (f) microshovel streaking through target droplet.

[6] J. T. Feddema and T. R. Christenson, "Parallel assembly of high aspect ratio microstructures," in SPIE Conference on Microrobotics and Micromanipulation, Boston, USA, September 1999, pp. 153-164.

[7] B. J. Nelson, Y. Zhou, and B. Vikramaditya, "Sensor-based microassembly of hybrid mems devices," IEEE J. of Control System, pp. 35-45, December 1998

[8] S. Yu and B. J. Nelson, "Autonomous injection of biological cells using visual servoing," The International Journal of Robotics Research (IJRR), vol. 21, no. 10-11, pp. 861-868, October-November 2002.

[9] H.-Y. Chan and W. J. Li, "A thermally actuated polymer micro robotic gripper for manipulation of biological cells," in Proc. IEEE International Conference on Robotics and Automation, September 2003, pp. 288-293.

[10] F. Arai, T. Sakami, H. Maruyama, A. Ichikawa, and T. Fukuda, "Minimally invasive micromanipulation of microbe by laser trapped micro tools," in Proc. IEEE International Conference on Robotics and Automation, Washington, DC, May 2002, pp. 1937-1942.

[11] R. E. Thorne, Z. Stum, J. Kmetko, K. O'Neill, and R. Gillilan, "Microfabricated mounts for high-throughput macromolecular cryocrystollagrophy," Journal of Applied Crystallography, no. 36, pp. 1455-1460, 2003.

[12] S. Haliyo, S. Regnier, and J.-C. Guinot, "[mü]mad, the adhesion based dynamic micro-manipulator," European Journal of Mechanics - A/Solids, vol. 22, no. 6, pp. 903-916, November 2003.

[13] A. D'Arcy, A. M. Sweeny, and A. Haber, "Using natural seeding material to generate nucleation in protein crystallization experiments," Acta Crystallographica D, vol. 59, no. 7, pp. 1343-1343, 2003.

[14] G. Flandin, F. Chaumette, and E. Marchand, "Eye-in-hand / eye-to-hand cooperation for visual servoing," in IEEE Int. Conf. on Robotics and Automation, San Francisco, CA, April 2000.

[15] A. Georgiev, P. K. Allen, and W. Edstrom, "Visually-guided protein crystal manipulation using micromachined silicon tools," in Proc. IEEE/RSJ Int. Conf. on Intelligent Robots and Systems, IROS'04, September 2004 pp. 236-241.

[16] N. Otsu, "A threshold selection method from gray-level histograms," IEEE Transactions on Systems, Man, and Cybernetics, vol. 9, no. 1, pp. 62-66, 1979. 